\title{
RANKING FOR PREHARVEST COTTONS BY USING MACHINE VISION
}

\author{
Ling Wang ${ }^{1}$, Changying $\mathrm{Ji}^{1, *}$ \\ ${ }^{1}$ College of Engineering, Nanjing Agricultural University, Nanjing, 210031, China \\ * Corresponding author, Address: Department of Agricultural Mechanization, College of \\ Engineering, Nanjing Agricultural University, 40 Dianjiangtai Road, Nanjing, 210031, \\ P.R. China,Tel: 13951994628, Email: chyji@njau.edu.cn
}

\begin{abstract}
In order to assess the quality of preharvest cottons objectively, ranking classifiers were designed based on machine vision technologies to grade preharvest cottons on dark background based on their sizes and colors. Experiments showed that the classifiers can classify preharvest cottons into seven grade categories with an accuracy of nearly $91.5 \%$.
\end{abstract}

Keywords: Preharvest Cottons, Grade, Machine vision, Classifier

\section{INTRODUCTION}

Over a long period, cottons have been mostly harvested in either manual or machinery way in China. The former is highly subjective, which is not accurate for grading, while the latter is not able to grade at all. It is necessary to come up with an approach to grade the preharvest cottons.

At present, although much research has been done on ginned cottons for grading outside and in by HVI equipment (Poceciun, 1999), little work has been done on un-ginned cottons as preharvest cottons for grading outside. This thesis presented the results of experiments in which classifiers was designed to sample the preharvest cottons and then grade them by employing machine vision and pattern recognition.

Wang, L. and Ji, C., 2008, in IFIP International Federation for Information Processing, Volume 259; Computer and Computing Technologies in Agriculture, Vol. 2; Daoliang Li; (Boston: Springer), pp. 1465-1469. 


\section{MATERIALS AND METHODS}

The samples were acquired by camera afield. A black board was placed behind each sample in the course of photo to segment from backdrop well. The total samples (402) were classified into seven grades ranged from 1 to 7 in manual, each of which contained 10, 28, 91, 67, 67, 69, 70.

\section{RESULTS AND DISCUSSIONS}

\subsection{Images segmentation}

Morphology operation, including dilation, erosion, opening, closing, tophat cutting, and bottom-hat cutting, is a shape-based technique of image processing (Gonzalez, 2002). Opening operation eroded original intensity images and then dilated eroded images with the same big structuring elements. And top-hat cutting images subtracted morphologically opened images from intensity images. Accordingly, closing operation dilated the original intensity image and then eroded the dilated image using the same small structuring element for both operations. And bottom-hat cutting images subtracted intensity images from morphologically closed images.

\subsubsection{Cottons with bracteoles segmentation from background}

Intensity images with brightened bracteoles were extracted by using intensity images plus adjusted top-hat cutting images, and then transformed into binary images based on Otsu's threshold. So cotton binary images with bracteoles were segmented from their background with noises (Figure 1).

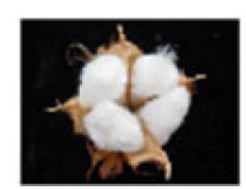

(a)

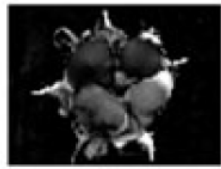

(b)

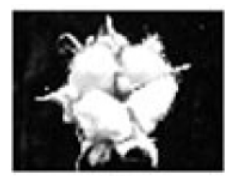

(c)

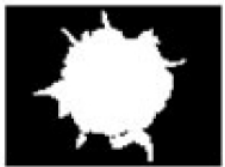

(d)

Figure 1. Cotton image segmentation from background
(a) Intensity image
(b) Top-hat cutting image
(c) Intensity image with brightened bracteoles
(d) Cotton binary image with bracteoles 


\subsubsection{Cottons segmentation from their bracteoles}

Intensity images with darkened bracteoles subtracted adjusted bottom-hat cutting images from intensity images, and then transformed into binary images based on Otsu's threshold. So cotton binary images were segmented from their bracteoles in turn because of bits and pieces of bracteoles being far small (Figure 2).

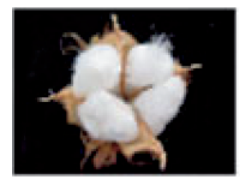

(a)

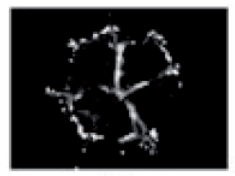

(b)

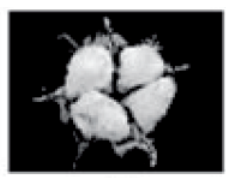

(c)

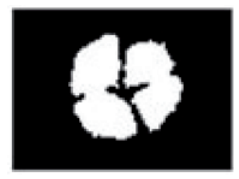

(d)

Figure 2. Cotton image segmentation from bracteoles

(a) Color image

(b) Bothat cutting image

(c) Intensity image with darkened bracteoles

(d) Cotton binary image without bracteoles

\subsection{Features selection}

According to Chinese government standards in letters in the purchase of preharvest cottons (Xiong, 2005), the primary factors for determining acceptability grades prior to purchase are external quality of cottons, including size, colors, textures, and impurities. Size and colors, implying textures and impurities respectively, were selected in this experiment.

Size (size) was calculated from the rate of the number of pixels of cotton with bracteoles to bracteoles. Much experiment results showed that hue of cotton is close to 10YR in Munsell Color Ring (Xiong, 1995), accordingly, and their color was only described by using saturation and intensity in HSI color space. So colors contained yellow degree $(Y d / y d)$ and white degree $(W d / w d)$, which were calculated from the mean value of saturation and intensity image of cotton with/without bracteoles, respectively. Furthermore, white contrast $(W c / w c)$ was calculated from the standard deviation of intensity image of cotton with/without bracteoles.

Minimum information redundancy among the features is a major goal. According to Chinese government standards, the relationship of size and $Y d / y d$ is negative, similarly, size and $W c / w c$, but that of size and $W d / w d$ is positive. Table 1 showed that $w d$ was invalid because of sunlight.

Table1. The relationship between size and colors

\begin{tabular}{c|cccccc}
\hline & Yd & Wd & Wc & yd & wd & wc \\
\hline Size & -0.668 & 0.574 & -0.617 & -0.332 & -0.125 & -0.245 \\
\hline
\end{tabular}




\subsection{Classifiers design}

\subsubsection{Grades clustering}

Different inspectors would result in different grades. Heinemann studied mushroom whereby the disagreement between inspectors varied from $14 \%$ to $34 \%$ compared to less than $20 \%$ of the machine vision system (Heinemann, 1994). It is necessary to cluster preharvest cottons into 7 grades based on machine vision, and a compact cluster was recovered by k-Means algorithm in 6-dimension vector space, including size, $Y d, W d, W c, y d, w c$. Table 2 showed that the relationships between clustering grades and 6 features are more approximate and no feature dominates others, which verify the clustering results.

Table 2. The relationship between grades and features

\begin{tabular}{lcccccc}
\hline Grades & Size & Yd & Wd & Wc & yd & wc \\
\hline Inspector & -.737 & 0.602 & -.264 & 0.629 & 0.310 & 0.507 \\
Clustering & -.822 & 0.860 & -.623 & 0.649 & 0.621 & 0.366 \\
\hline
\end{tabular}

\subsubsection{Principal component analysis}

The optimality of Principal component analysis (PCA) with respect to the minimum MSE will lead to excellent information packing properties. PCA was performed to generate optimally uncorrelated features, keep the size of classifiers as small as possible and increase the generalization capabilities. In this experiment, the 6 correlated features of size, $Y d, W d, W c, y d, w c$ were reduced to only 2 orthonormal eigenvectors, i.e. the first and the second principal component (Prin. $1 \& 2$ ), which corresponded to nearly $78 \%$ in cumulative variation.

\subsubsection{Linear discriminant}

Fisher linear discriminant function is an optimal linear classifier by minimizing MSE, which had two inputs (i.e. Prin.1\&Prin.2) and one output (i.e. clustered grades). Fisher's linear discriminant functions were computed based on within-groups covariance in SPSS (Table 3).

Table 3. Fisher's classification functions coefficients

\begin{tabular}{lrrrrrrr}
\hline Principal & \multicolumn{7}{c}{ Function } \\
\cline { 2 - 8 } components & 1 & \multicolumn{1}{c}{2} & \multicolumn{1}{c}{3} & \multicolumn{1}{c}{5} & \multicolumn{1}{c}{6} & \multicolumn{1}{c}{7} \\
\hline Const. & -25.723 & -7.778 & -4.769 & -1.922 & -5.351 & -4.974 & -8.767 \\
Prin. 1 & -16.441 & -7.940 & -5.573 & -1.220 & -.516 & 4.914 & 8.680 \\
Prin. 2 & 1.894 & 1.735 & -1.263 & 1.489 & -4.806 & 3.142 & -2.340 \\
\hline
\end{tabular}


The apparent reclassification results varied from $64 \%$ to $98 \%$ with the average of $91.5 \%$ by re-substitution method, which gave some indication on the consistency of machine vision decision and verified the clustering results (Table 4).

Table 4. Reclassification results by using re-substitution (\%)

\begin{tabular}{cccccccc}
\hline Grouped & \multicolumn{7}{c}{ Group } \\
\cline { 2 - 8 } cases & 1 & 2 & 3 & 4 & 5 & 6 & 7 \\
\hline 10 & 90 & 10 & 0 & 0 & 0 & 0 & 0 \\
39 & 0 & 64 & 23 & 13 & 0 & 0 & 0 \\
53 & 0 & 4 & 90 & 6 & 0 & 0 & 0 \\
92 & 0 & 1 & 0 & 98 & 1 & 0 & 0 \\
64 & 0 & 0 & 5 & 0 & 95 & 0 & 0 \\
89 & 0 & 0 & 0 & 4 & 0 & 96 & 0 \\
55 & 0 & 0 & 0 & 0 & 9 & 0 & 91 \\
\hline
\end{tabular}

\section{CONCLUSION}

This thesis indicates the applications of machine vision technology involving size and colors grading of preharvest cottons. A prototype automated preharvest cottons classifier based on previously developed algorithm for size and colors assessment is successfully developed and tested. Reclassification results of cotton grade categories with an average accuracy of nearly $96 \%$, and the results demonstrate the validity of the clustering results previously. Hence, machine vision systems helps standardizing and quantifying the inspection process of field preharvest cottons by promoting grading consistency and objectivity.

\section{REFERENCES}

Gonzalez, R.C. and Woods, R.E. Digital Image Processing, Second Edition. [M]. Beijing: Publishing House of Electronics Industry. 2002, 7:519-569.

Heinemann, P.H., Hughes, R., Morrow, C.T., Sommer III, H.J., Beelman, R.B., \& West, P.J. (1994). Grading of mushrooms using a machine vision system. Transactions of the ASAE, 37(5), 1671-1677.

Poceciun V.V., Temkin B., Ethridge M.D., Hequet E. Computerized collection and analysis of HVI data. U.S.A. Orlando, National Cotton Council of America, Proceedings Beltwide Cotton Conference, 1999.

Xiong Z.W. Study on the fiber quality and the classification of color grades of China's cotton.

Master Paper of China Agricultural University, 2005:10-25.

Xiong Z.W. Cotton Color Features [J]. China Cotton, 1995, 22 (4): 39-40. 